\title{
Factores asociados a la sobrecarga en el cuidador primario. Medellín, 2017
}

\author{
Factors associated with overloading in the primary caregiver. Medellin, 2017
}

Beatriz Torres-Avendaño1* orcid.org/0000-0002-7953-3525

Maite Catalina Agudelo-Cifuentes' orcid.org/0000-0003-1501-9452

Ángela María Pulgarin-Torres' orcid.org/0000-0002-5302-7501

Dedsy Yajaira Berbesi-Fernández' orcid.org/0000-0002-6586-4120

1 Facultad de Enfermería, Universidad CES. Medellín, Colombia

\section{Resumen}

Introducción: El aumento de personas dependientes conlleva a la necesidad de cuidadores que asuman tareas básicas de cuidado; la responsabilidad de cuidar es asumida principalmente por familiares y el cuidado constante repercute en cambios de índole personal, familiar, laboral y social. Objetivo: Identificar los principales factores sociodemográficos y del cuidado, asociados a la sobrecarga en el cuidador primario de la ciudad de Medellín para el año 2017. Materiales y métodos: Estudio transversal, cuantitativo, de fuente de información primaria obtenida por encuestas telefónicas. La muestra fue de 340 cuidadores. El consentimiento fue verbal. Resultados: El 92,6\% de los cuidadores eran mujeres, la edad promedio fue de 57 años; el 53,8\% percibía su salud como regular o mala. La prevalencia de sobrecarga fue 39,7\% y las características asociadas fueron la mala percepción de salud, el abandono de actividades y el no recibir capacitación previa al cuidado. Conclusiones: El cuidador es un sujeto con derechos que requiere atención en programas de prevención de riesgos que se derivan del cuidado. Es necesario brindar educación y acompañamiento a las personas encargadas del cuidado para que se empoderen de su salud.

Palabras clave: Cuidadores; dependencia; agotamiento emocional. (Fuente: DeCS, Bireme).

\begin{abstract}
Introduction: The increase in dependent people leads to the need for caregivers who assume basic tasks of care. The responsibility to care is assumed mainly by family members and the constant care impacts on changes of personal, family, labor and social nature. Objective: To identify the main sociodemographic and care factors associated with the overload in the primary caregiver in the city of Medellín during 2017. Materials and methods: A cross-sectional, quantitative study was made, which primary information source was obtained by telephone surveys. The sample was 340 caregivers and the consent was verbal. Results: $92.6 \%$ of the caregivers were women whose average age was 57; 53.8\% perceived their health as regular or bad. The prevalence of overload was $39.7 \%$ and the associated characteristics were poor perception of health, abandonment of activities and not receiving pre-care training. Conclusions: The caregiver is a subject with rights that requires care in risk prevention programs that derive from care. It is necessary to provide education and accompaniment to caregivers so they are empowered of their health.
\end{abstract}

Key words: Caregivers; dependency; burnout, professional. (Source: DeCS, Bireme).

\footnotetext{
*Autor de correspondencia

Beatriz Torres Avendaño

e-mail: btorres@ces.edu.co
} 


\section{Introducción}

Los cambios epidemiológicos como el envejecimiento poblacional, el aumento de enfermedades crónicas y discapacidades físicas y psíquicas, generan un incremento en el número de personas con dependencia; aunque ésta no es una condición exclusiva de la población adulta mayor, su incidencia incrementa en edades avanzadas, por lo tanto, es un fenómeno que se ve influenciado con el cambio demográfico y se constituye como un reto en temas de políticas sociales, para hacer frente a las necesidades que se derivan del envejecimiento de la población(1). El aumento de personas dependientes, conlleva a la necesidad de cuidadores que asuman tareas básicas de cuidado y que ayuden a disminuir el impacto negativo que tiene la dependencia sobre la calidad de vida y bienestar(2). La responsabilidad de cuidar es asumida principalmente por familiares, quienes se constituyen como la principal fuente de apoyo(1).

La dependencia es la incapacidad funcional para realizar las actividades básicas de la vida diaria, que conlleva a necesidad de asistencia(2,3); el cuidado de la persona dependiente puede estar delegado a un profesional que no pertenece al grupo familiar y que es remunerado por asumir este rol, llamado "cuidador formal"(4); sin embargo el cuidado de la persona con algún grado de discapacidad recae principalmente en la familia(1,5) o alguna persona cercana, llamada "cuidador informal"(4), quien no recibe ningún tipo de remuneración(6,7). Aproximadamente en el $70 \%$ de los casos, las personas con discapacidad son cuidadas por familiares, y en el $80 \%$ son las mujeres quienes brinda este cuidado, principalmente en edades avanzadas(1); este rol es asumido por voluntad, necesidad $u$ obligación(6-8), sin recibir capacitación y sin tener experiencia. Estas personas no solo asumen la responsabilidad del cuidado, sino también de las decisiones que involucran el bienestar y el cubrimiento de las necesidades básicas del sujeto de cuidado ${ }^{(9,10)}$.

Este cuidador principal, que es quien está al frente del cuidado y destina gran parte de su tiempo a esta labor. La responsabilidad diaria y constante del cuidado de una persona dependiente, puede desencadenar cambios de índole personal, familiar, laboral y social y afectar directa o indirectamente la salud física, mental y bienestar subjetivo del cuidador(11).

En cuanto a salud mental, la responsabilidad de cuidar de una persona dependiente puede generar ansiedad, depresión, estrés y sobrecarga(12,13). La sobrecarga del cuidador es un estado de agotamiento emocional, estrés y cansancio, que afecta directamente las actividades de ocio, relaciones sociales, libertad y equilibrio mental(7,12-14); es el grado en el cual el cuidador percibe la influencia negativa del cuidado, en diferentes aspectos de su vida como en la salud mental y física, la interacción social y su economía(15). Hay diferentes características asociadas a la sobrecarga, entre las cuales se encuentra el grado de dependencia para realizar actividades de la vida diaria del sujeto de cuidado y el número elevado de horas al cuidado(6).

El presente estudio se justifica en la necesidad de identificar las principales características de los cuidadores de personas con dependencia de la ciudad de Medellín, para aportar con evidencia científica a tomadores de decisiones, resaltando la necesidad de planes y programas que promuevan el bienestar y apoyo a personas que asumen el rol de cuidador; se planteó como objetivo identificar los principales factores sociodemográficos y del cuidado, asociados a la sobrecarga en el cuidador primario de la ciudad de Medellín para el año 2017.

\section{Materiales y métodos}

Estudio transversal, cuantitativo, con intención analítica, de fuente de información primaria obtenida a partir de encuestas telefónicas realizadas a cuidadores primarios de la ciudad de Medellín en el año 2017. La población de referencia, fue tomada a partir de un marco muestral consultado en fuentes oficiales de la ciudad de Medellín, donde se encontraban registrados los cuidadores primarios; se tuvo como criterios de inclusión que fueran cuidadores primarios, determinado a partir de la 
percepción del propio cuidador o de otro familiar cercano, al dedicar la mayor parte de su tiempo al cuidado de la persona con dependencia; otro criterio de inclusión fue que habitaran en la ciudad de Medellín. Se excluyeron los cuidadores que manifestaron tener inconvenientes para contestar la encuesta telefónica. En caso de detectarse más de un cuidador, se asumió como principal aquel que reportó un mayor acompañamiento. Para el cálculo de la muestra se tomó como referencia el número de cuidadores de la ciudad de Medellín y una sobrecarga del cuidador primario del 25\%, obteniendo un tamaño muestral de 340 cuidadores, los cuales fueron seleccionados del marco muestral por selección aleatoria simple, utilizando muestreo con reemplazo para sustituir a aquellos que no residían en la vivienda al momento de la encuesta o que hubieran fallecido.

La encuesta telefónica fue la técnica de recolección de información, esta fue realizada a los cuidadores que dieron su consentimiento informado verbal luego de explicarse el objetivo del estudio, la confidencialidad de los datos y la participación voluntaria. En los casos en los cuales el cuidador principal no se encontró en la vivienda al momento de la llamada, se programó una nueva llamada en horario y fecha en el cual el encuestador pudiera ser atendido. Se realizaron preguntas sobre características sociodemográficas y preguntas relacionadas con el cuidado para establecer algunas características que pudieran estar asociadas a la sobrecarga. Se utilizó la escala Zarit que permite cuantificar el grado de sobrecarga que tienen los cuidadores de personas dependientes(16), fue creada en 1983 por Zarit S, Orr N y Zarit J; ha sido ampliamente utilizada a nivel nacional e internacional y validada en diversos idiomas incluidos el español(17). Permite cuantificar el grado de sobrecarga del cuidador por medio de 22 preguntas tipo Likert que evalúan tres principales factores: efecto del cuidado, carga interpersonal y expectativas de autoeficacia. Los 22 ítems se plantean en forma de afirmaciones que describen cómo se sienten los cuidadores, en cada pregunta el cuidador debe indicar la frecuencia con que se siente identificado con ésta, utilizando una escala que va de 0 a 4 (0 es nunca, 1 es rara vez, 2 es algunas veces, 3 es bastantes veces, y 4 indica casi siempre). Los puntajes obtenidos se suman para obtener un puntaje final que oscila entre 0 y 88; cuando el rango de respuestas total es menor de 46 indica no sobrecarga, de 46 a 56 sobrecarga leve, mayor de 56 sobrecarga intensa(15,17,18).

Se usó el cuestionario Katz, para determinar el grado de dependencia del sujeto de cuidado entendida como la incapacidad de velar por sí mismo en el desarrollo de las actividades básicas de la vida diaria (19,20); éste evalúa seis funciones sociobiológicas que son: bañarse, vestirse, usar el retrete, moverse, control de micción o defecación y alimentación; es un cuestionario de 6 ítems dicotómicos que clasifica la persona en 8 posibles niveles que va desde independiente en todas sus funciones, hasta dependiente en al menos dos funciones(19,20).

Para controlar el sesgo de información se realizó estandarización de los encuestadores, a partir de los resultados obtenidos en la prueba piloto que se hizo al cuestionario, la cual se realizó con diez cuidadores y se pudo establecer el tiempo de diligenciamiento, la reacción de los cuidadores con algunas preguntas, evaluar el desempeño del encuestador y la forma adecuada de dirigir las preguntas.

No se tomaron datos personales de los encuestados, pero para hacer control de calidad se guardó registro del teléfono para poder llamarlo cuando fuese necesario. Para el procesamiento de la información se utilizó el programa estadístico SPSS versión 21. Se realizó análisis univariado para caracterizar los cuidadores por medio de frecuencias absolutas y relativas. Para identificar las características asociadas a la sobrecarga del cuidador se realizó análisis bivariado, calculando razones de prevalencias, intervalos de confianza del 95\% y valores de $\mathrm{p}$, tomando como significativos los inferiores a 0,05 ; para el modelo ajustado se ingresaron las variables que cumplieron criterio de hosmer-lemeshow con valores de $p$ por debajo de 0,25, este análisis multivariado se realizó por medio de regresión logística binaria. 


\section{Consideraciones éticas}

En cuanto a los aspectos éticos, se contó con aprobación del Comité de Ética de la Universidad CES y según la Resolución 8430 de 1993 esta investigación se clasifica sin riesgo, dado que emplea como técnica de recolección la encuesta telefónica en la cual no se realizó ninguna intervención de las variables a analizar ni se trataron aspectos sensitivos de la conducta(21).

\section{Resultados}

92,6\% de los cuidadores eran mujeres; la edad promedio fue de 57 años (DE 13,74), siendo la edad más frecuente 60 años. El 72,8\% de los cuidadores pertenecían a estrato socioeconómico bajo (estrato 1 y 2), el 27,2\% a estrato medio (estrato 3 y 4). En cuanto al estado civil, el 56,8\% de los cuidadores se encontraban sin pareja (soltero, viudo, separado). Se preguntó acerca del número de hijos que tenían los cuidadores, encontrándose que el 32,6\% tenía 4 hijos o más, el 50,3\% tenía entre 1 y 3 , y el 17,1\% no tenía ninguno.

De los cuidadores encuestados, el $10,9 \%$ no tenían ningún nivel educativo, el $44,8 \%$ alcanzaron estudios de primaria, el 38,1\% de secundaria y solo el 6,2\% realizaron algún estudio técnico, tecnológico o profesional. En cuanto al estado de salud del cuidador de acuerdo con su percepción, se encontró que el $46,2 \%$ percibía su salud como buena, el $49,7 \%$ como regular y el $4,1 \%$ como mala.

En relación al número de personas que tenía el cuidador a cargo, se observó que el 10,0\% era responsable de tres o más personas, hallándose cuidadores a cargo de hasta 7 personas, el 22,9\% cuidaba a dos personas y el 67,1\% era únicamente responsable del cuidado de una persona.

En cuanto al cuidado brindado, el $81,8 \%$ de los encuestados indicaron ser únicos cuidadores y el $18,2 \%$ restante cuentan con el apoyo de otras personas. El 98,8\% asume este rol los 7 días de la semana y el 1,2\% entre 5 y 6 días; en relación al número de horas diarias que brinda el cuidado, el $95,6 \%$ se encarga de esto las 24 horas del día y el $4,4 \%$ cuida entre 3 y 23 horas diarias. El $78,2 \%$ de los cuidadores manifestó haber abandonado algunas de sus actividades deportivas, culturales, recreativas o laborales, para asumir el rol de cuidador. Únicamente el $5,0 \%$ recibe alguna retribución económica o en especie por el cuidado que brinda. De los cuidadores que fueron encuestados, el 55,9\% informaron haber recibido alguna vez inducción o capacitación sobre como brindar el cuidado.

\section{Cuidadores con sobrecarga:}

Se identificó una prevalencia de sobrecarga intensa de $19,4 \%$, sobrecarga leve de $20,3 \%$ y el $60,3 \%$ no tenían sobrecarga. De los cuidadores con sobrecarga leve o intensa, el 93,3\% eran mujeres, sin presentar diferencias estadísticas significativas por sexo $(\mathrm{p}=0,694)$; la proporción por edad tampoco mostró diferencias significativas, el $50,4 \%$ de los cuidadores con sobrecarga eran menores de 60 años y el $49,6 \%$ de 60 años o más. El 69,1\% habitaban viviendas de estrato bajo, sin ser significativa la diferencia con los de estrato medio ( $\mathrm{p}=0,256)$; el $57,8 \%$ se encontraban sin pareja $(p=0,760)$; el $51,1 \%$ tenían entre 1 y 3 hijos, sin encontrarse diferencia significativa con aquellos que no tenían hijos $(\mathrm{p}=0,525)$.

En cuanto a la percepción de salud, se encontró que el 59,3\% de los cuidadores con sobrecarga la consideraba regular, el 7,4\% mala y el 33,3\% manifestaron percibir un buen estado de salud, presentándose diferencias estadísticas significativas $(\mathrm{p}=0,001)$ (Tabla 1$)$.

En cuanto a las características relacionadas con el cuidado, el $88,1 \%$ de los cuidadores con sobrecarga era único cuidador y el 11,9\% tenía ayuda de otras personas, siendo significativa la diferencia ( $p=0,013)$; el 99,3\% brindaba el cuidado los siete días de la semana y solo el 0,7\% lo cuidaba entre cinco y seis días presentar diferencia significativa ( $p=0,480) ;$ el $99,3 \%$ asume el rol de cuidador las 24 horas del día y el $0,7 \%$ cuidaba entre 3 y 23 horas, siendo significativa la diferencia ( $\mathrm{p}=0,005)$. El 91,9\% de los cuidadores con sobrecarga manifestaron haber abandonado actividades para dedicarse al cuidado $\quad\left(X^{2}=24,380, \quad p<0,001\right)$, el $98,5 \%$ 
manifestó no recibir ninguna retribución económica ni en especie $\left(X^{2}=5,836, p=0,016\right)$; el
$51,1 \%$ recibieron inducción o capacitación sobre como brindar el cuidado (Tabla 2).

Tabla 1. Características sociodemográficas de cuidadores primarios de sujetos de cuidado. Medellín, 2017.

\begin{tabular}{|c|c|c|c|c|c|c|c|}
\hline & & \multicolumn{2}{|c|}{ Con sobrecarga } & \multicolumn{2}{|c|}{ Sin sobrecarga } & \multirow{2}{*}{ Ji cuadrado } & \multirow{2}{*}{$\mathbf{p}$} \\
\hline & & $N$ & $\%$ & $N$ & $\%$ & & \\
\hline \multirow{2}{*}{ Sexo } & Hombre & 9 & 6,7 & 16 & 7,8 & \multirow{2}{*}{0,155} & \multirow{2}{*}{0,694} \\
\hline & Mujer & 126 & 93,3 & 189 & 92,2 & & \\
\hline \multirow{2}{*}{ Edad } & Menor de 60 años & 68 & 50,4 & 105 & 51,7 & \multirow{2}{*}{0,059} & \multirow{2}{*}{0,807} \\
\hline & De 60 años o más & 67 & 49,6 & 98 & 48,3 & & \\
\hline \multirow{2}{*}{ Estrato } & Bajo (1-2) & 76 & 69,1 & 125 & 75,3 & \multirow{2}{*}{1,289} & \multirow{2}{*}{0,256} \\
\hline & Medio (3-4) & 34 & 30,9 & 41 & 24,7 & & \\
\hline \multirow{2}{*}{ Estado civil } & Con pareja & 57 & 42,2 & 90 & 43,9 & \multirow{2}{*}{0,094} & \multirow{3}{*}{0,760} \\
\hline & Sin pareja & 78 & 57,8 & 115 & 56,1 & & \\
\hline \multirow{3}{*}{ Hijos } & Sin hijos & 26 & 19,3 & 32 & 15,6 & \multirow{3}{*}{1,290} & \\
\hline & Entre 1 y 3 hijos & 69 & 51,1 & 102 & 49,8 & & \multirow[t]{2}{*}{0,525} \\
\hline & 4 o más hijos & 40 & 29,6 & 71 & 34,6 & & \\
\hline \multirow{3}{*}{$\begin{array}{l}\text { Percepción } \\
\text { estado salud }\end{array}$} & Buena & 45 & 33,3 & 112 & 54,6 & \multirow{3}{*}{17,994} & \multirow{3}{*}{$<0,001$} \\
\hline & Regular & 80 & 59,3 & 89 & 43,4 & & \\
\hline & Mala & 10 & 7,4 & 4 & 2,0 & & \\
\hline
\end{tabular}

Tabla 2. Características relacionadas con el cuidado, según cuidadores primarios.

\begin{tabular}{|c|c|c|c|c|c|c|c|}
\hline & & \multicolumn{2}{|c|}{ Con sobrecarga } & \multicolumn{2}{|c|}{ Sin sobrecarga } & \multirow{2}{*}{ Ji cuadrado } & \multirow{2}{*}{$\mathbf{p}$} \\
\hline & & $n$ & $\%$ & $n$ & $\%$ & & \\
\hline \multirow[t]{3}{*}{ Personas a cargo del cuidado } & Una persona & 84 & 62,2 & 144 & 70,2 & \multirow{3}{*}{2,418} & \multirow{3}{*}{0,298} \\
\hline & Dos personas & 35 & 25,9 & 43 & 21,0 & & \\
\hline & Tres o más personas & 16 & 11,9 & 18 & 8,8 & & \\
\hline \multirow[t]{2}{*}{ Cuidadores } & Dos o más cuidadores & 16 & 11,9 & 46 & 22,4 & \multirow{2}{*}{6,119} & \multirow{2}{*}{0,013} \\
\hline & Único cuidador & 119 & 88,1 & 159 & 77,6 & & \\
\hline \multirow[t]{2}{*}{ Días al cuidado } & Cinco-seis & 1 & 0,7 & 3 & 1,5 & & \multirow{2}{*}{$0,480^{*}$} \\
\hline & Siete & 134 & 99,3 & 202 & 98,5 & & \\
\hline \multirow[t]{2}{*}{ Horas diarias al cuidado } & 3-23 horas & 1 & 0,7 & 14 & 6,8 & & \multirow{2}{*}{$0,005^{*}$} \\
\hline & 24 horas & 134 & 99,3 & 191 & 93,2 & & \\
\hline \multirow[t]{2}{*}{ Abandono de actividades } & No & 11 & 8,1 & 63 & 30,7 & \multirow{2}{*}{24,380} & \multirow{2}{*}{$<0,001$} \\
\hline & $\mathrm{Si}$ & 124 & 91,9 & 142 & 69,3 & & \\
\hline \multirow[t]{2}{*}{ Retribución económica } & $\mathrm{Si}$ & 2 & 1,5 & 15 & 7,3 & \multirow{2}{*}{5,836} & \multirow{2}{*}{0,016} \\
\hline & No & 133 & 98,5 & 190 & 92,7 & & \\
\hline \multirow[t]{2}{*}{ Recibió capacitación } & $\mathrm{Si}$ & 69 & 51,1 & 121 & 59,0 & \multirow[b]{2}{*}{2,067} & \multirow{2}{*}{0,150} \\
\hline & No & 66 & 48,9 & 84 & 41,0 & & \\
\hline
\end{tabular}

*Prueba exacta de Fisher

La característica que mostró mayor asociación a la sobrecarga del cuidador primario fue el tiempo al día dedicado al cuidado, se encontró que es 9 veces más probable la sobrecarga en las personas que se encargan del cuidado las 24 horas del día, que en aquellos que dedican menos horas diarias (RP crudo=9,82; IC95\% 1,2875,59). La percepción del cuidador sobre su estado de salud tiene fuerte asociación con la sobrecarga, siendo mayor la probabilidad de sobrecarga en quienes tienen mala percepción de su estado de salud, que en quienes la percepción es buena (RP crudo=6,22; IC95\% 1,85-20,87) y regular (RP crudo=2,24; IC95\% 1,41-3,54). Los cuidadores que abandonaron algunas de sus actividades para dedicarse al cuidado, tuvieron 5 veces la sobrecarga de quienes no abandonaron sus actividades (RP crudo=5,00; IC95\% 2,529,91); los que no recibían ninguna retribución económica, tuvieron 5 veces la probabilidad de 
sobrecarga, de quienes si la recibían (RP crudo=5,25; IC95\% 1,81-23,34). La probabilidad de sobrecarga en los cuidadores únicos fue dos veces la de los cuidadores que contaban con ayuda (RP crudo=2,15; IC95\% 1,16-3,99).

Al realizar el modelo ajustado por estas variables incluyendo la variable capacitación, dado el criterio Hosmer-Lemeshow, se encontró que la probabilidad de sobrecarga continúa siendo mayor en los cuidadores únicos, que en aquellos que cuentan con ayuda; sin embargo, ésta asociación pierde significancia estadística al ajustar por las demás variables (RP ajustado =1,84; IC95\% 0,95-3,57). Brindar el cuidado las 24 horas del día también perdió significancia estadística (RP ajustado =5,18; IC95\% 0,5945,42 ), al igual que el no recibir retribución económica por el cuidado (RP =3,67; IC95\% 0,77-17,85); el abandono de actividades conservó su significancia estadística $(\mathrm{p}<0,001)$ (Tabla 3).

Tabla 3. Fuerza de asociación entre características del cuidado y la sobrecarga en el cuidador primario

\begin{tabular}{|c|c|c|c|c|c|c|}
\hline Características & RP crudo & IC 95\% & $\mathbf{p}$ & RP ajustado & IC 95\% & $\mathbf{p}$ \\
\hline Percepción estado salud & & & $<0,001$ & & & 0,004 \\
\hline Buena & 1 & -- & & 1 & -- & \\
\hline Regular & 2,24 & $1,41-3,54$ & 0,001 & 2,02 & $1,24-3,28$ & 0,005 \\
\hline Mala & 6,22 & $1,85-20,87$ & 0,003 & 4,57 & $1,24-16,81$ & 0,022 \\
\hline \multicolumn{7}{|l|}{ Cuidadores } \\
\hline Dos o más cuidadores & 1 & -- & \multirow{2}{*}{0,015} & 1 & -- & \multirow{2}{*}{0,070} \\
\hline Único cuidador & 2,15 & $1,16-3,99$ & & 1,84 & $0,95-3,57$ & \\
\hline \multicolumn{7}{|l|}{ Horas diarias al cuidado } \\
\hline 3-23 horas & 1 & -- & \multirow{2}{*}{0,028} & 1 & -- & \multirow{2}{*}{0,138} \\
\hline 24 horas & 9,82 & $1,28-75,59$ & & 5,18 & $0,59-45,42$ & \\
\hline \multicolumn{7}{|l|}{ Abandono de actividades } \\
\hline No & 1 & -- & \multirow{2}{*}{$<0,001$} & 1 & -- & \multirow{2}{*}{$<0,001$} \\
\hline $\mathrm{Si}$ & 5,00 & $2,52-9,91$ & & 4,11 & $1,99-8,47$ & \\
\hline \multicolumn{7}{|l|}{ Retribución económica } \\
\hline $\mathrm{Si}$ & 1 & -- & \multirow{2}{*}{0,029} & 1 & -- & \multirow{2}{*}{0,103} \\
\hline No & 5,25 & $1,81-23,34$ & & 3,67 & $0,77-17,85$ & \\
\hline \multicolumn{7}{|l|}{ Capacitación } \\
\hline $\mathrm{Si}$ & 1 & -- & \multirow{2}{*}{0,151} & 1 & -- & \multirow{2}{*}{0,028} \\
\hline No & 1,38 & $0,89-2,13$ & & 1,72 & $1,06-2,79$ & \\
\hline
\end{tabular}

El modelo permitió observar que la probabilidad de sobrecarga en los cuidadores con mala percepción de su estado de salud disminuyó respecto al primer modelo, pero se mantuvo significativa, siendo cuatro veces mayor a la probabilidad de sobrecarga de quienes perciben su salud como buena (RP $=4,57$; IC95\% 1,2416,81).

Los cuidadores que abandonaron actividades para dedicarse al cuidado tuvieron cuatro veces la probabilidad de sobrecarga en relación con aquellos que no tuvieron que abandonar sus actividades diarias (RP =4,11; IC95\% 1,99-8,47); esta probabilidad también disminuyó, pero mantuvo su significancia estadística. En cuanto a recibir capacitación o inducción acerca de cómo realizar un cuidado adecuado, que inicialmente no había mostrado asociación significativa, al ser ajustado por las demás variables, mostró un aumento en la fuerza de la asociación, identificándose que la probabilidad de sobrecarga en quienes no recibieron capacitación fue 1,7 veces la probabilidad de quienes si recibieron alguna inducción $(\mathrm{RP}=1,72$; IC95\%1,06-2,79) (Figura 1). 


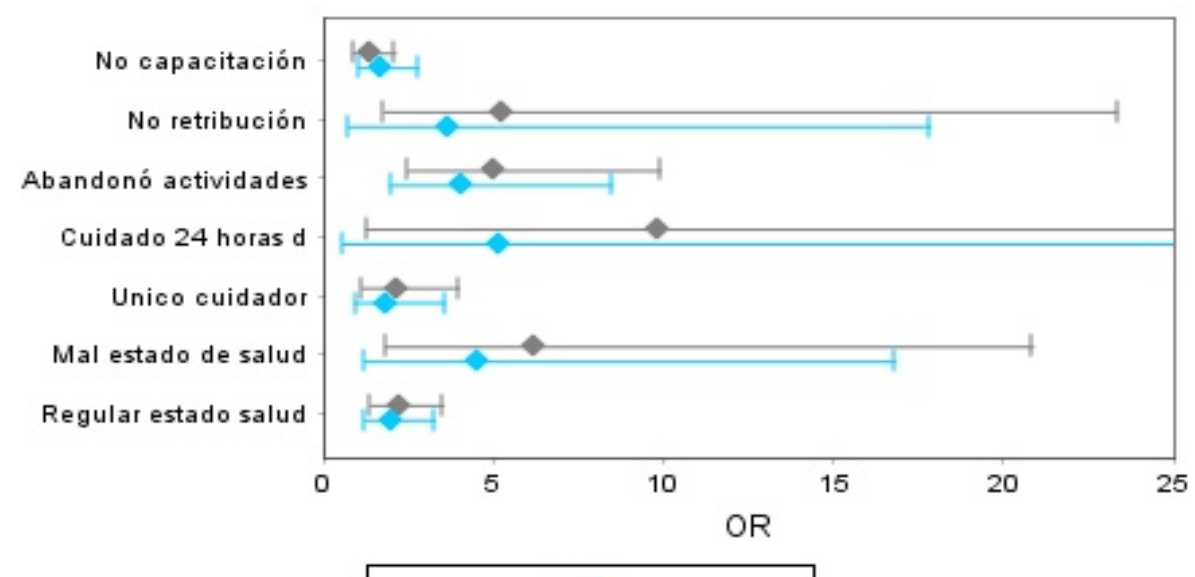

OR crudo $\square$ OR ajustado

Figura 1. Factores asociados a la sobrecarga del cuidador primario de sujetos de cuidado

\section{Discusión}

El presente estudio permitió caracterizar de acuerdo con factores sociodemográficos a los cuidadores primarios de personas sujeto de cuidado en la ciudad de Medellín, encontrándose que son principalmente mujeres, y en su mayoría próximas a llegar a la vejez quienes asumen este rol; hallazgo que difiere a lo reportado en un estudio realizado en Valledupar, Colombia, en el cual se identificó que los cuidadores eran principalmente hombres, representando estos un $94 \%{ }^{(22)}$

La prevalencia encontrada de sobrecarga en el cuidador fue de $39,7 \%$, con una fuerte asociación con la mala percepción del estado de salud. Son las familias y principalmente las mujeres quienes asumen el cuidado de las personas dependientes, teniendo además otras funciones como ser madre de familia y amas de casa(23). Encontrar mayoría de mujeres que asumen el cuidado y que se encuentran en edades avanzadas, también ha sido reportado en otros estudios, donde los hallazgos han reflejado que las mujeres en edad avanzada se están dedicando al cuidado de sus familiares, cuando ellas mismas deben ser objeto de cuidado y acciones preventivas $(3,4,6,13,24,25)$.

La responsabilidad de cuidar a una persona dependiente involucra esfuerzo, tiempo y en ocasiones trae para el cuidador repercusiones a largo plazo que son negativas para su salud y calidad de vida, lo cual afecta tanto al cuidador como del sujeto de cuidado(3). La persona que asume el rol de cuidador tiene que modificar su estilo de vida para dedicar parte de su tiempo a estar al pendiente de las necesidades de otra persona(3); en la presente investigación se identificó un alto porcentaje de cuidadores que aparte del rol de cuidador, debían dividir sus actividades diarias para responder a las necesidades de otras personas con alguna dependencia. El tener ayuda de un familiar o persona cercana, podría convertirse en un factor protector ante los eventos negativos como enfermedades, estrés y sobrecarga que se derivan del cuidado de una persona dependiente $(3,7,13)$. Entre las actividades que debe dejar de lado un cuidador para asumir su rol, se encuentran las laborales o las que le generan ingresos económicos, lo cual se constituye como un factor de disminución de capacidad económica, considerando que son muy pocas las personas dependientes que cuentan con un cuidador remunerado para apoyar sus actividades básicas de la vida diaria(26); en el presente estudio, el porcentaje de cuidadores que manifestaron recibir alguna remuneración por el cuidado, fue muy bajo.

Este estudio identificó que cuatro de cada diez cuidadores tenía sobrecarga, similar a la reportada en otras investigaciones(23), sin embargo, en un estudio realizado para el año 2010 en la ciudad de Medellín, fue reportada una prevalencia de sobrecarga del cuidador primario 
de adultos mayores de 12,1\%(27). Se identificó asociación significativa entre la sobrecarga y la mala percepción del estado de salud, este resultado es similar a lo reportado en otros estudios(27); en las personas que desempeñan el rol de cuidadores, es frecuente la aparición de problemas de salud tanto física como mental, principalmente síntomas depresivos, muchas de las cuales pueden ser provocadas por las labores propias del cuidado y por el descuido propio de los cuidadores quienes dejan a un lado el autocuidado y sus propias necesidades; además también respecto a esto es importante considerar que la edad de los cuidadores se acerca a la vejez, lo cual genera un incremento en los problemas de salud y en la necesidad de cuidado $(3,7,8,26)$.

Investigaciones han identificado que la sobrecarga en el cuidador se encuentra asociada al número de horas diarias que se dedica al cuidado(3,6,8,27), esto se vio reflejado en el presente estudio que identificó asociación significativa entre la sobrecarga y brindar el cuidado las 24 horas del día, se encontró que casi la totalidad de cuidadores se ocupaba del cuidado los 7 días de la semana de manera permanente y durante todo el día.

El envejecimiento poblacional, es considerado un logro para la salud pública, el cual trae grandes retos a la sociedad, entre los cuales se encuentra hacer frente al aumento de personas dependientes que requieren de cuidado, que con frecuencia es brindado de manera informal por familiares y $\operatorname{cercanos}^{(1)}$; el cuidado permanente de una persona que requiere atención y que por lo general cuenta con algún nivel de dependencia, ocasiona a largo plazo repercusiones negativas para quien asume el rol de cuidador, hecho que a su vez puede afectar el bienestar de la persona que es cuidada, dado que un cuidado exitoso depende en gran medida de la salud física y mental del cuidador; por lo cual el autocuidado de quien asume el papel de cuidador, se convierte en eje fundamental de esta relación de dependencia(22).

\section{Conclusiones}

En la ciudad de Medellín la prevalencia de sobrecarga del cuidador es alta, afectando esto principalmente su percepción de estado de salud; son las mujeres quienes en mayor medida se encuentran asumiendo el rol de cuidadores. El papel que desempeña el cuidador primario es indispensable para las personas que tienen algún tipo de discapacidad o limitación que le impide valerse por sí misma; sin embargo, el cuidador también es un sujeto con derechos, que requiere atención en programas de prevención de riesgos que se derivan del cuidado dado que se ven constantemente sometidos a estrés y presión. La sociedad tiene el reto de disminuir la carga del cuidador primario, apoyándose con programas, planes y proyectos encaminados a mejorar la calidad de vida de los cuidadores; se debe brindar educación y acompañamiento a las personas encargadas del cuidado de familiares o personas cercanas, para que sean ellos quienes se empoderen de su salud, refuercen acciones de autocuidado y prevención de consecuencias negativas derivadas de su rol. Con este estudio se concluye que el abandono de actividades para dedicarse al cuidado, la no retribución económica y la falta de inducción o capacitación son características asociadas que requieren ser focalizadas a la hora de plantear acciones y programas que busquen el mejoramiento de la calidad de vida tanto del cuidador como del sujeto de cuidado

\section{Limitaciones}

Considerando el diseño del estudio, no se analizó causalidad, por lo cual los resultados únicamente pueden interpretarse como factores asociados y no factores de riesgo. También se contó con la limitación de que los datos fueron tomados por autoreporte y que la realización de la encuesta fue telefónica.

\section{Conflicto de intereses}

Ninguno declarado por los autores. 


\section{Referencias}

1. Pérez-Salanova M, Yanguas-Lezaun JJ. Dependencia, personas mayores y familias. De los enunciados a las intervenciones. An Psicol. 1998;14(1):95-104.

2. Alegre-Escolano A, Ayuso-Gutiérrez M, Guillén-Estany M, Monteverde Verdenelli M, Pociello García E. Tasa de dependencia de la población española no institucionalizada y criterios de valoración de la severidad. Rev Esp Salud Pública. 2005;79:351-63.

3. Flores GE, Rivas RE, Seguel PF. Nivel de sobrecarga en el desempeño del rol del cuidador familiar de adulto mayor con dependencia severa. Cienc Enferm. 2012;18(1):29-41.

4. Cerquera M, Granados J, Buitrago M. Sobrecarga en cuidadores de pacientes con demencia tipo Alzheimer. Psychol Av Discip. 2012;6(1):35-45.

5. Vélez JM, Berbesí D, Cardona D, Segura A, Ordóñez J. Validación de escalas abreviadas de zarit para la medición de síndrome del cuidador primario del adulto mayor en Medellín. Aten Primaria. 2012;44(7):411-6.

6. Rodríguez-González AM, Rodríguez-Míguez E, DuartePérez A, Díaz-Sanisidro E, Barbosa-Álvarez Á, Clavería A. Estudio observacional transversal de la sobrecarga en cuidadoras informales y los determinantes relacionados con la atención a las personas dependientes. Aten Primaria. 2017;49(3):156-65.

7. Espinoza K, Jofre V. Sobrecarga, apoyo social y autocuidado en cuidadores informales. Cienc Enferm. 2012;18(2):23-30.

8. Sánchez RT, Molina EM, Gómez OR. Intervenciones de enfermería para disminuir la sobrecarga en cuidadores: un estudio piloto. Rev Cuid. 2016;7(1):1171.

9. Tripodoro V, Veloso V, Llanos V. Sobrecarga del cuidador principal de pacientes en cuidados paliativos. Argumentos: revista de crítica social. 2015;17:307-330.

10. Rosina E, Sawada N, Ferreira J. Asociación entre la incapacidad funcional del anciano y la sobrecarga del cuidador familiar. Rev Latino-Am Enfermagen. 2013;21(5):1-8.

11. Bermejo F. Aspectos familiares y sociales de los pacientes con demencia. Segunda Edición. Madrid: Ediciones Díaz de Santos; 2004.

12. Méndez L, Giraldo O, Aguirre D, Lopera F. Relación entre ansiedad, depresión, estrés y sobrecarga en cuidadores familiares de personas con demencia tipo alzheimer por mutación e280a en presenilina 1 . Rev Chil Neuropsicol. 2006;5(2):137-45.

13. Carrillo GM, Chaparro L, Sánchez B. Carga del cuidado en cuidadores familiares de personas con enfermedad crónica en la región pacífica colombiana. Cienc Enferm. 2014;20(2):83-91.

14. Martínez FE. Recuperación de la salud del cuidador principal, en proyecto sociocultural. Los Sitios, Centro Habana. Enferm Glob. 2012;11(25):92-103.

15. Prieto E, Arias N, Villanueva Y, Jiménez A. Síndrome de sobrecarga del cuidador en familiares de pacientes geriátricos atendidos en un hospital de segundo nivel. Med Int Méx. 2015;31:660-8.
16. Álvarez L, González AM, Muñoz P. El cuestionario de sobrecarga del cuidador de Zarit: Cómo administrarlo e interpretarlo. Gac Sanit. 2008;22(6):618-9.

17. Zarit S, Orr N, Zarit J. The hidden victims of Alzheimer's disease: families under stress. New York: University Press; 1985.

18. Diéguez C, Ponce S, Lorca B. Factores de riesgo de la dependencia funcional en las personas mayores chilenas y consecuencias en el cuidado informal = Risk factors of dependency of Chilean older adults and consequences on informal caregiving. América Lat Hoy. 2015;71:17-36.

19. Katz S, Down TD, Cash HR, et al. Progress in development of the index of ADL. Gerontologist, 1.970;10(1):20-30.

20. Katz S, Ford AB, Moskowitz RW, et al. Studies of illness in the age: the index of ADL a standarized measure of biological and psycosocial function. JAMA. 1963;185: 914-919.

21. República de Colombia. Ministerio de Salud. Resolución Nº08430 de 1993. Bogotá: MinSalud; 1993.

22. Rojas-Picón Y, Montalvo-Prieto AA, Díaz-Gómez AA. Afrontamiento y adaptación de cuidadores familiares de pacientes sometidos a procedimientos cardioinvasivos. Universidad y Salud. 1 de mayo de 2018;20(2):131-8.

23. Yonte F, Urien Z, Martín M, Montero R. Sobrecarga del cuidador principal. Rev. enferm. 2010;2(1):59-69.

24. Jofré V, Sanhueza O. Evaluación de la sobrecarga de cuidadoras/es informales. Cienc Enferm. 2010;16(3):111-20.

25. Larrinoa P, Martínez S, Carrasco M. Autopercepción del estado de salud en familiares cuidadores y su relación con el nivel de sobrecarga. Psicothema. 2011;23(3):388-93.

26. Timonet E, Morales M, Canca C, Rivas F, Mesa R, Sepúlveda J, et al. Calidad de vida relacionada con la salud y sobrecarga de cuidadores de pacientes ingresados con insuficiencia cardiaca. Enferm En Cardiol. 2013;58-59(1, 2):50-5.

27. Cardona D, Segura A, Berbesí D, Agudelo M. Prevalencia y factores asociados al síndrome de sobrecarga del cuidador primario de ancianos. Rev Fac Nac Salud Pública. 2013;31(1):30-9. 